Disponible en ligne sur

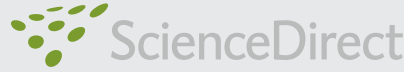

www.sciencedirect.com
Elsevier Masson France

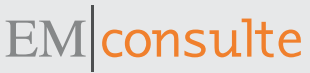

www.em-consulte.com

\title{
Le couplage ventriculoartériel : du concept aux applications cliniques
}

\section{Ventriculo-arterial coupling: From concepts to clinical applications}

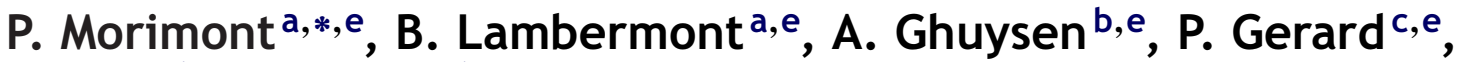 \\ P. Kolh ${ }^{d, e}$, V. D'Orio ${ }^{b, e}$
}

a Unité de soins intensifs médicaux, centre hospitalier universitaire de Liège, domaine universitaire du Sart Tilman, 4000 Liège, Belgique

b Service des urgences, centre hospitalier universitaire de Liège, Liège, Belgique

c Département de mathématique, université de Liège, Liège Belgique

d Service de chirurgie cardiovasculaire et thoracique, centre hospitalier universitaire de Liège, Liège, Belgique

' Centre de recherche en hémodynamique (Hémoliège), université de Liège, Liège, Belgique

Disponible sur Internet le 14 février 2009

\section{MOTS CLÉS}

Couplage ventriculoartériel ;

Hémodynamique ;

Insuffisance

ventriculaire

\section{KEYWORDS}

Ventriculo-arterial coupling;

Hemodynamics;

Ventricular failure
Résumé L'interaction entre le ventricule et le réseau vasculaire est un déterminant majeur de la performance cardiaque globale, particulièrement en présence d'une insuffisance ventriculaire préalable. L'évaluation du couplage ventriculoartériel grâce à la mesure de l'élastance ventriculaire, comme reflet de la contractilité et de l'élastance artérielle, en tant qu'indice de post-charge, permet de quantifier cette interaction. Des travaux récents illustrent l'intérêt clinique de ce concept. Jusqu'à présent, son utilisation restait toutefois marginale en raison de la nécessité de recourir à des mesures invasives et complexes. Le développement des techniques d'imagerie non invasive et de traitement des signaux permet actuellement d'envisager l'utilisation de ce concept en pratique clinique courante.

(c) 2009 Société de réanimation de langue française. Publié par Elsevier Masson SAS. Tous droits réservés.

Summary Ventriculo-arterial interaction is a major determinant of global cardiac performance, particularly in pre-existing ventricular failure. Ventriculo-arterial coupling concept quantifies this interaction by assessing ventricular and arterial elastances, representing ventricular contractility and post-charge respectively. Recent investigations showed clinical interest of this concept. However, its application has always been restricted by the need of invasive and complex measurements. Because of non-invasive imaging and signal analysis developments, this concept tends to be used in clinical practice.

(c) 2009 Société de réanimation de langue française. Published by Elsevier Masson SAS. All rights reserved.

\section{Introduction}

* Auteur correspondant.

Adresse e-mail : ph.morimont@chu.ulg.ac.be (P. Morimont).
La performance ventriculaire dépend de l'interaction permanente entre la pompe cardiaque et le réseau vas-

1624-0693/\$ - see front matter @ 2009 Société de réanimation de langue française. Publié par Elsevier Masson SAS. Tous droits réservés. doi:10.1016/j.reaurg.2009.01.013 
culaire d'aval. Le ventricule gauche est en effet une pompe chargée de fournir à la masse sanguine une énergie suffisante pour pouvoir circuler au sein du réseau vasculaire systémique et atteindre l'oreillette droite. Les caractéristiques ventriculaires intrinsèques fixent les conditions initiales de l'écoulement vasculaire. En corollaire, par ses propriétés élastiques et résistives, le réseau vasculaire systémique a la valeur d'une contrainte physique qui fixe les caractéristiques de l'environnement dans lequel la pompe doit opérer. Dès lors, l'évaluation de la performance cardiovasculaire requiert d'abord de caractériser chacun des constituants: le réseau vasculaire systémique et la contractilité ventriculaire gauche, tout en tenant compte du caractère spécifiquement pulsatile de la circulation. Ces deux composants opèrent de manière simultanée, de telle sorte que leur interaction, c'est-à-dire leur couplage, détermine le volume d'éjection systolique et la pression d'éjection. Ce concept de couplage ventriculoartériel est également applicable au versant pulmonaire pour apprécier l'importance de l'interaction entre le ventricule droit et le système artériel pulmonaire.

Bien que le concept de couplage ventriculoartériel soit connu depuis de nombreuses années, son application clinique a toujours été limitée par la lourdeur et le caractère invasif des mesures nécessaires à sa détermination. L'objectif de cet article est de rappeler les bases théoriques du couplage ventriculoartériel et de montrer la possibilité de son application clinique.

\section{Aspects théoriques du couplage ventriculoartériel}

Le concept de couplage ventriculoartériel, développé par Sunagawa et al., assimile les propriétés du ventricule et de l'arbre vasculaire à deux chambres élastiques dont le comportement peut être traduit en termes d'équations algébriques simples [1,2].

\section{Propriétés ventriculaires}

Le système ventriculaire peut être décrit comme une chambre à élastance variable et caractérisé par l'élastance télésystolique ventriculaire gauche(Ees), donnée par l'expression suivante:

$\operatorname{Ees}=\left[\frac{P(t)}{\left(V(t)-V^{\circ}\right)}\right]_{\max }$

où $\mathrm{P}(\mathrm{t})$ et $\mathrm{V}(\mathrm{t})$ sont les pressions et volumes ventriculaires gauches respectivement et $\mathrm{V}$ le volume mort. Ees définit la contractilité ventriculaire et est indépendante des conditions de charge, elle est déterminée par la pente de la relation entre la pression de fin de systole et le volume à différents niveaux de charge (ESPVR) (Fig. 1). L'équation de cette droite d'élastance ventriculaire est donnée par:

Pes $=\operatorname{Ees}\left(\right.$ Ves $\left.-V^{\circ}\right)=\operatorname{Ees}\left(\right.$ Ved $\left.-V^{\circ}-S V\right)$

où Pes et Ves sont la pression et le volume de fin de systole, Ved le volume fin de diastole et SV le volume éjecté. $V^{\circ}$ est l'intersection de cette droite avec l'axe des abscisses.

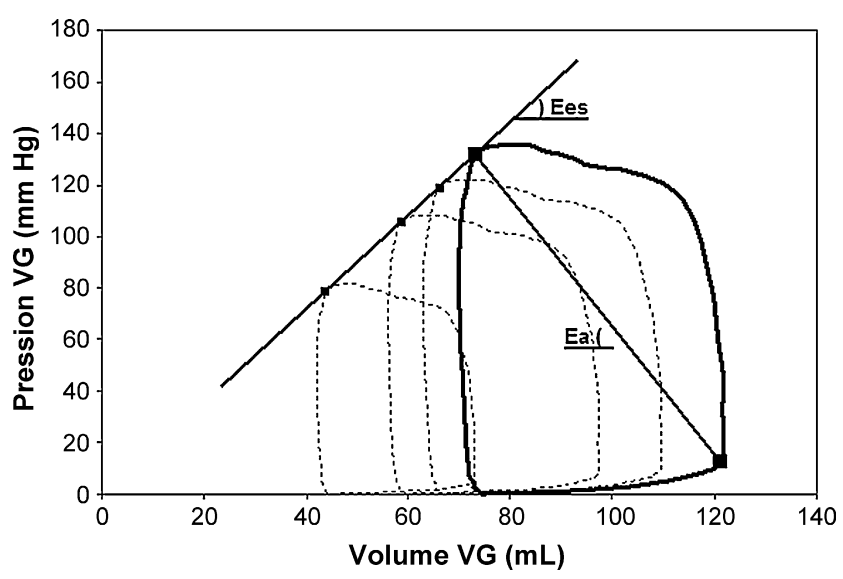

Figure 1 L'élastance télésystolique ventriculaire gauche (Ees) représente la contractilité ventriculaire. Elle est déterminée par la pente de la relation entre la pression de fin de systole et le volume (ESPVR), à différents niveaux de charge. L'élastance artérielle (Ea) est définie par l'équation $\mathrm{Ea}=\mathrm{Pes} / \mathrm{SV}$ où Pes est la pression de fin de systole et SV le volume éjecté.

La détermination d'Ees nécessite donc une modification de précharge pour obtenir différents points de la droite ESPVR. Une telle modification de précharge étant difficilement réalisable en pratique clinique, plusieurs méthodes alternatives ont été développées pour éviter de recourir à une manipulation du retour veineux. La méthode dite single beat proposée par Takeuchi et al. consiste à ajuster une sinusoïde sur l'onde de pression ventriculaire [3]. Le maximum de cette sinusoïde correspond à la pression maximale qui serait développée par un cycle non éjectant (Fig. 2). Le point correspondant à ce cycle non éjectant dans l'espace pression-volume (PV) est défini par cette pression calculée et le volume télédiastolique. La droite d'élastance maximale (ESPVR) est obtenue en traçant la tangente à la boucle PV passant par ce point (Fig. 2).

\section{Propriétés vasculaires}

L'impédance d'entrée de l'aorte ascendante fournit la description la plus complète de la charge hydraulique à laquelle le ventricule est confronté car elle caractérise complètement le réseau vasculaire [4,5]. Elle tient compte en effet du caractère pulsatile et des réflexions d'onde dans tout l'arbre vasculaire. Cette impédance est un nombre complexe, déterminé dans le domaine fréquentiel [6]. L'inconvénient de son analyse dans le domaine fréquentiel est qu'elle ne peut alors être confrontée directement aux propriétés ventriculaires qui sont, elles, exprimées dans le domaine temporel. L'utilisation d'un modèle Windkessel à 3 (ou 4) éléments, analogie électrique du système cardiovasculaire, permet de calculer séparément les différents composants de cette impédance (la résistance des gros vaisseaux, la résistance périphérique et la compliance) (Fig. 3) [7]. Les différents éléments du modèle Windkessel peuvent alors être exprimés dans le domaine temporel sous forme d'une élastance par la relation suivante [8]:

$\mathrm{Ea}(\mathrm{WK})=\frac{\mathrm{RT}}{\left[\mathrm{ts}+\left(1-e^{-\mathrm{td} /} \tau\right)\right]}$ 

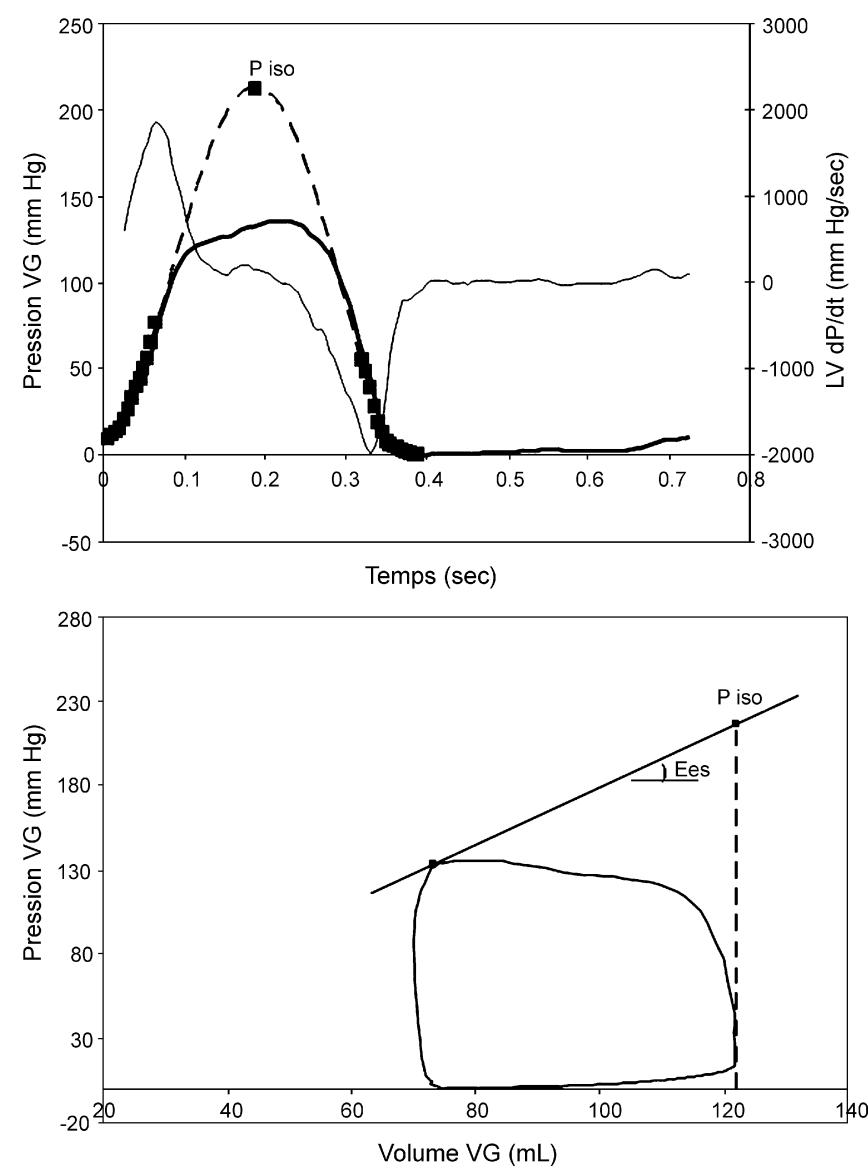

Figure 2 Une sinusoïde est ajustée sur la courbe de pression ventriculaire avant $\mathrm{dP} / \mathrm{dt}_{\max }$ et après $\mathrm{dP} / \mathrm{dt}_{\min }$ (points en carré gras). Le maximum de cette courbe définit une pression maximale qui serait développée par un cycle non éjectant (Piso) au volume télédiastolique (Ved) (graphe du dessus). Ved et Piso définissent donc un point de la droite ESPVR dans l'espace PV. La pente de la tangente à la boucle PV passant par ce point donne Ees (graphe du dessous).

où RT est la résistance vasculaire totale, égale à la somme de la résistance caractéristique du système (gros vaisseaux) (R1) et de la résistance périphérique (R2). $\tau$ est la constante de temps de décroissance diastolique de l'onde de pression égale au produit de RT et de la compliance de l'arbre vasculaire $(C)$. ts et td sont respectivement les temps de systole et de diastole. Cette élastance partage donc les mêmes unités que l'élastance ventriculaire (Ees) et caractérise complètement les propriétés du système vasculaire.

Il a été démontré par Sagawa et al. que Ea peut aussi être simplement calculée par le rapport de Pes/SV où Pes est la pression télésystolique du ventricule gauche [8]. La droite d'élastance artérielle dans l'espace PV est donc donnée par:

Pes $=$ EaSV

Chez l'homme, Kelly et al. ont montré que cette relation était applicable au versant systémique [9]. Sur le versant pulmonaire, nous avons montré, chez l'animal, que cette équation pouvait être appliquée à condition de tenir compte de la pression d'aval du circuit pulmonaire (qui n'est pas négligeable, contrairement au versant systémique), c'est-à-dire en soustrayant la pression de l'oreillette gauche à Pes [10].

\section{Le couplage ventriculoartériel}

La performance de la pompe ventriculaire peut ainsi être décrite par les deux droites d'élastance définies par les Eq. (1) et (3). Le point de fonctionnement, aussi appelé couplage, du système correspond à l'intersection de ces deux droites et fixe le volume éjecté correspondant à Pes (Fig. 1) :

$\mathrm{SV}=\frac{\operatorname{Ees}\left(\mathrm{Ved}-V^{\circ}\right)}{(\operatorname{Ees}+\mathrm{Ea})}$

\section{Aspects énergétiques}

Toute source d'énergie est adaptée à sa charge (matching) lorsqu'une partie maximale de l'énergie est transférée de la première à la seconde $[1,11]$.

La Fig. 4 illustre que l'aire délimitée par le segment systolique de la boucle PV, la droite ESPVR et la relation PV télédiastolique (PVA) représente la somme du travail mécanique externe ( $S W=$ aire de la boucle $P V)$ généré par le ventricule et d'une énergie potentielle, emmagasinée à la fin de la systole (EP) $[12,13]$. Le rapport de SW/PVA quantifie l'efficience du transfert énergétique de l'énergie totale vers le travail mécanique externe SW [11,14].

Le rapport Ees/Ea est appelé index de couplage ventriculoartériel. Il est mathématiquement démontré que si ce rapport approche une valeur de 2, l'efficience du système est alors maximale, tandis qu'une valeur proche de 1 favorise un SW maximum. Enfin, une valeur sous l'unité est évocatrice de découplage $[1,14]$. Suga et al. ont par ailleurs démontré l'existence d'une relation linéaire entre la consommation d'oxygène par le myocarde $\left(\mathrm{MVO}_{2}\right)$ et PVA : $M \mathrm{O}_{2}=\mathrm{a} \cdot \mathrm{PVA}+\mathrm{b}$ où $\mathrm{a}$ et $\mathrm{b}$ sont respectivement la pente
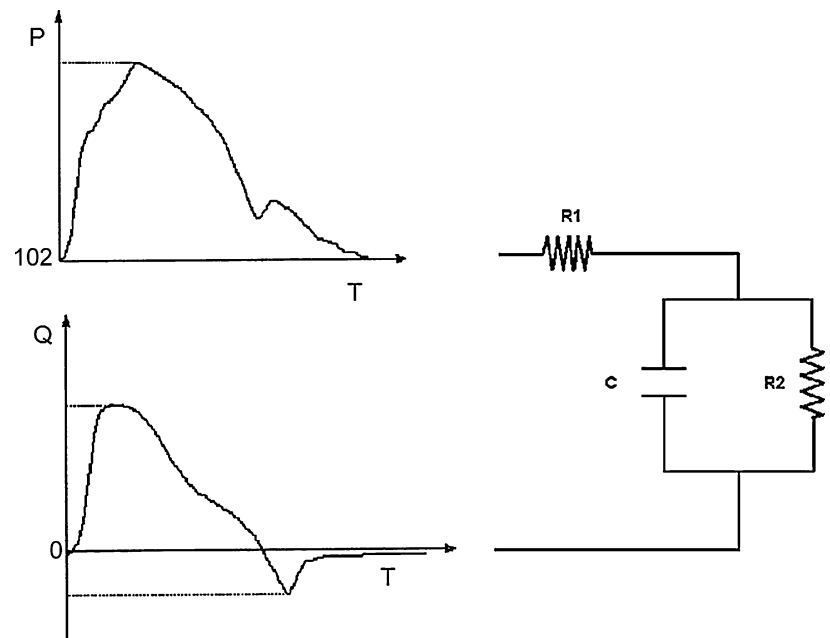

Figure 3 Représentation d'un modèle Windkessel à trois éléments ( $R 1$ : impédance caractéristique; $R 2$ : résistance périphérique; $C$ : compliance). Les différents éléments du modèle sont calculés par l'ajustement aux données observées de la relation existant entre le débit $(\mathrm{Q})$ et la pression aortique $(\mathrm{P})$, par analogie au circuit électrique. 


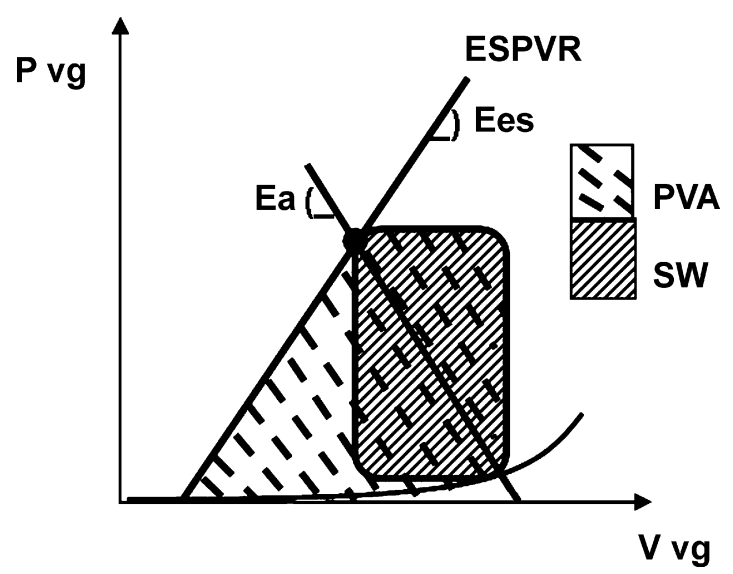

Figure 4 Dans l'espace pression (Pvg) - volume ventriculaire gauche $(\mathrm{Vvg})$, représentation du travail mécanique externe (SW) et de l'énergie potentielle (PVA) générés par un cycle cardiaque. Le rapport SW/PVA est l'efficience mécanique cardiaque et dépend du rapport de l'élastance télésystolique ventriculaire (pente de la droite des points de fin de systole (ESPVR) et de l'élastance artérielle: Ees/Ea.

de la relation et l'ordonnée à l'origine de cette relation [15].

\section{Intérêt clinique du couplage ventriculoartériel}

Une des premières publications mettant en évidence l'importance clinique du couplage ventriculoartériel est celle de Binkley et al. $[16,17]$. Ces auteurs ont étudié la variation du volume d'éjection systolique en réponse à l'administration de doses croissantes de dobutamine chez des patients présentant une cardiomyopathie congestive dilatée. Ils ont observé que, malgré une sollicitation pharmacologique identique de la réserve contractile du ventricule gauche, chez certains patients (répondeurs), le volume éjecté augmentait significativement, alors que chez les autres (non-répondeurs), celui-ci ne changeait pas. Les auteurs ont constaté que, chez les répondeurs, l'effet inotrope positif de la dobutamine s'accompagnait d'une modification du spectre d'impédance aortique (réduction de la résistance caractéristique, augmentation de la compliance et diminution des phénomènes de réflexion d'ondes) correspondant à une diminution de l'élastance artérielle de manière à faciliter le couplage ventriculoartériel.

En théorie, une évaluation objective du couplage ventriculoartériel permettrait d'optimiser le traitement hémodynamique [18]. En effet, l'interaction ventriculoartérielle est perçue de manière intuitive au travers de la fraction d'éjection ventriculaire gauche et des résistances vasculaires systémiques. On administre des vasodilatateurs et des inotropes positifs en présence d'une hypertension avec fraction d'éjection ventriculaire gauche effondrée. Au contraire, on privilégie les vasopresseurs face à un patient en choc septique afin d'augmenter les résistances vasculaires systémiques et le recours à des agents inotropes sont indiqués si une dysfonction systolique du ventricule gauche lui est associée.

Toutefois, il est établi que la fraction d'éjection ne reflète pas nécessairement le niveau de contractilité ven- triculaire et que la post-charge ventriculaire ne peut être réduite à la résistance vasculaire moyenne $[19,20]$. De plus, cette dernière ne tient compte ni du caractère pulsatile des ondes de pression et de débit, ni des phénomènes de réflexions d'ondes alors que ces derniers influencent de manière significative la charge à laquelle le ventricule est confronté au cours de l'éjection $[21,22]$. Il est donc utile de caractériser complètement les propriétés ventriculaires et artérielles de manière à disposer d'un seul index permettant d'évaluer leur interaction et donc l'adéquation du traitement hémodynamique. En outre, la détermination du couplage ventriculoartériel permet d'apprécier l'efficience du transfert d'énergie du myocarde vers le système artériel. Au plan expérimental, il est bien établi que le couplage ventriculoartériel est altéré dans l'infarctus du myocarde, l'embolie pulmonaire et le choc septique [23-26]. La restauration d'un couplage ventriculoartériel optimal par voie pharmacologique est possible dans certaines de ces pathologies [23-26]. Le choc cardiogénique se traduit par un «découplage ventriculoartériel »: le maintien de la pression artérielle requiert une élévation de post-charge face à une chute du débit cardiaque. L'utilisation d'un ballon intra-aortique de contre-pulsion permet la restauration d'un certain degré de couplage [27]. La resynchronisation ventriculaire améliore également le couplage ventriculoartériel dans l'insuffisance cardiaque [28].

\section{Aspects pratiques du couplage ventriculoartériel}

L'application pratique du concept de couplage ventriculoartériel au niveau clinique s'est toujours heurtée à la difficulté d'acquérir des boucles PV ventriculaires nécessaires à la détermination de l'élastance ventriculaire (Ees) et de l'élastance artérielle (Ea).

\section{Volume ventriculaire}

Chez l'animal, on utilise classiquement des cathéters à conductance ou des capteurs à microcristaux. En bref, les cathéters à conductance mesurent le volume ventriculaire par la conductance qu'offre la masse sanguine du ventricule à un courant entre un pôle émetteur et plusieurs pôles récepteurs situés sur le même cathéter. De tels cathéters à destination clinique nécessitent un abord indépendant pour leur mise en place et leur diamètre est important [29]. Les capteurs à microcristaux mesurent par ultrasons la variation de distance entre plusieurs paires de capteurs positionnés directement sur le péricarde et en déduisent le volume [30]. Grâce au développement des techniques d'imagerie cardiaque non invasives telles que l'échocardiographie (transœsophagienne ou transthoracique) ou l'IRM et des logiciels de traitement de l'image, le volume ventriculaire peut actuellement être acquis de manière continue et de manière non invasive $[31,32]$.

\section{Pression ventriculaire}

L'acquisition de l'onde de pression ventriculaire nécessite la mise en place d'un cathéter approprié. Le signal des 
cathéters standards fluid filled, utilisés en clinique, est altéré par l'impédance de la colonne d'eau située entre l'extrémité du cathéter et le capteur, contrairement aux cathéters disposant d'un capteur en bout de sonde. Toutefois, moyennant l'usage d'un filtre numérique, le signal de pression des cathéters fluid filled est exploitable [33]. À partir de l'onde de pression fémorale, couramment utilisée pour le monitoring continu de la pression artérielle, il est actuellement possible de reconstituer fidèlement la partie systolique de l'onde de pression ventriculaire.

\section{Construction des boucles pression-volume (PV)}

Les signaux de pression et de volume acquis simultanément doivent être échantillonnés à la même fréquence et synchronisés. Les boucles PV sont alors construites point par point.

\section{Détermination d'Ees}

Il est nécessaire de modifier les conditions de pré- ou de post-charge pour obtenir une succession de plusieurs points de fin de systole et déterminer la pente de la droite ESPVR. Expérimentalement, une diminution de précharge est réalisée en gonflant un ballon dans la veine cave inférieure, ce qui est difficilement envisageable en pratique clinique. Certains auteurs utilisent des moyens pharmacologiques tels que le nitroprussiate ou la phényléphrine, d'autres une charge volumique ou encore un levé passif des jambes $[34,35]$. La méthode single beat ne nécessite pas de modification de précharge mais requiert la connaissance de la partie diastolique de l'onde de pression ventriculaire.

\section{Détermination d'Ea}

Ea est déterminée par le simple rapport de Pes/SV sur le versant systémique ou (Pes - Pog)/SV sur le versant pulmonaire. De manière plus complexe, la connaissance de l'onde de débit aortique ou artérielle pulmonaire en combinaison avec l'onde de pression aortique ou pulmonaire permet de calculer Ea par l'analyse du modèle Windkessel et de ses éléments.

\section{Conclusion}

Le couplage ventriculoartériel est un élément déterminant de la performance cardiaque. Il est bien établi que la simple estimation de la fraction d'éjection ventriculaire et des résistances vasculaires ne permet pas de déterminer la performance ventriculaire ni d'apprécier l'efficience énergétique cardiovasculaire. Grâce à l'évolution des techniques d'imagerie non invasive et des logiciels de traitement des signaux, l'évaluation du couplage ventriculoartériel tend à devenir accessible en routine et pourrait aider l'intensiviste à adapter et optimiser le traitement selon les conditions hémodynamiques.

\section{Conflits d'intérêts}

Aucun.

\section{Références}

[1] Kass DA, Kelly RP. Ventriculo-arterial coupling: concepts, assumptions, and applications. Ann Biomed Eng 1992;20: 41-62.

[2] Sunagawa K, Sagawa K, Maughan WL. Ventricular interaction with the loading system. Ann Biomed Eng 1984;12:163-89.

[3] Takeuchi M, Igarashi Y, Tomimoto S, Odake M, Hayashi T, Tsukamoto T, et al. Single-beat estimation of the slope of the end-systolic pressure-volume relation in the human left ventricle. Circulation 1991;83:202-12.

[4] Nichols WW, Conti CR, Walker WE, Milnor WR. Input impedance of the systemic circulation in man. Circ Res 1977;40:451-8.

[5] Nichols WW, Pepine CJ, Geiser EA, Conti CR. Vascular load defined by the aortic input impedance spectrum. Fed Proc 1980;39:196-201.

[6] Maughan WL, Sunagawa K, Sagawa K. Effects of arterial input impedance on mean ventricular pressure-flow relation. Am J Physiol 1984;247:H978-83.

[7] Burkhoff D, Alexander Jr J, Schipke J. Assessment of Windkessel as a model of aortic input impedance. Am J Physiol 1988;255:H742-53.

[8] Sagawa K, Maughan WL, Suga H, Sunagawa K. Cardiac contraction and the pressure-volume relationship. New York: Oxford University Press; 1988.

[9] Kelly RP, Ting CT, Yang TM, Liu CP, Maughan WL, Chang MS, et al. Effective arterial elastance as index of arterial vascular load in humans. Circulation 1992;86:513-21.

[10] Morimont P, Lambermont B, Ghuysen A, Gerard P, Kolh P, Lancellotti $P$, et al. Effective arterial elastance as an index of pulmonary vascular load. Am J Physiol Heart Circ Physiol 2008;294:H2736-42.

[11] Gibbs CL. Cardiac energetics. Physiol Rev 1978;58:174-254.

[12] Burkhoff D, Sagawa K. Ventricular efficiency predicted by an analytical model. Am J Physiol 1986;250:R1021-7.

[13] Sunagawa K, Maughan WL, Sagawa K. Optimal arterial resistance for the maximal stroke work studied in isolated canine left ventricle. Circ Res 1985;56:586-95.

[14] Sarnoff SJ, Case RB, Welch Jr GH, Braunwald E, Stainsby WN. Performance characteristics and oxygen debt in a nonfailing, metabolically supported, isolated heart preparation. Am J Physiol 1958;192:141-7.

[15] Suga H, Igarashi Y, Yamada O, Goto Y. Mechanical efficiency of the left ventricle as a function of preload, afterload, and contractility. Heart Vessels 1985;1:3-8.

[16] Binkley PF, Van Fossen DB, Nunziata E, Unverferth DV, Leier $\mathrm{CV}$. Influence of positive inotropic therapy on pulsatile hydraulic load and ventricular-vascular coupling in congestive heart failure. J Am Coll Cardiol 1990;15:1127-35.

[17] Binkley PF, Van Fossen DB, Haas GJ, Leier CV. Increased ventricular contractility is not sufficient for effective positive inotropic intervention. Am J Physiol 1996;271:H1635-42.

[18] Lambermont B, D'Orio V. The role of right ventricularpulmonary arterial coupling to differentiate between effects of inotropic agents in experimental right heart failure. Crit Care Med 2006;34:2864-5.

[19] Naeije R. Pulmonary vascular resistance. A meaningless variable? Intensive Care Med 2003;29:526-9.

[20] Robotham JL, Takata M, Berman M, Harasawa Y. Ejection fraction revisited. Anesthesiology 1991;74:172-83.

[21] Curtis SL, Zambanini A, Mayet J, Thom SA, Foale R, Parker KH, et al. Reduced systolic wave generation and increased peripheral wave reflection in chronic heart failure. Am J Physiol Heart Circ Physiol 2007;293:H557-62.

[22] Westerhof BE, Guelen I, Stok WJ, Wesseling KH, Spaan JA, Westerhof $\mathrm{N}$, et al. Arterial pressure transfer characteristics: effects of travel time. Am J Physiol Heart Circ Physiol 2007; 292:H800-7. 
[23] Ghuysen A, Lambermont B, Dogne JM, Kolh P, Tchana-Sato V, Morimont P, et al. Effect of BM-573 [N-terbutyl-N'-[2-(4'methylphenylamino)-5-nitro-benzenesulfonyl]urea], a dual thromboxane synthase inhibitor and thromboxane receptor antagonist, in a porcine model of acute pulmonary embolism. J Pharmacol Exp Ther 2004;310:964-72.

[24] Kolh P, Lambermont B, Ghuysen A, D’Orio V, Gerard P, Morimont $P$, et al. Alteration of left ventriculo-arterial coupling and mechanical efficiency during acute myocardial ischemia. Int Angiol 2003;22:148-58.

[25] Lambermont B, Kolh P, Ghuysen A, Moonen M, Morimont P, Gerard $P$, et al. Effect of hemodiafiltration on pulmonary hemodynamics in endotoxic shock. Artif Organs 2003;27:1128-33.

[26] Lambermont B, Kolh P, Ghuysen A, Segers P, Dogne JM, Tchana-Sato V, et al. Effect of a novel thromboxane A2 inhibitor on right ventricular-arterial coupling in endotoxic shock. Shock 2004;21:45-51.

[27] Marchionni N, Fumagalli S, Baldereschi G, Di Bari M, Fantini F. Effective arterial elastance and the hemodynamic effects of intraaortic balloon counterpulsation in patients with coronary heart disease. Am Heart J 1998;135:855-61.

[28] Steendijk P, Tulner SA, Bax JJ, Oemrawsingh PV, Bleeker GB, van Erven $L$, et al. Hemodynamic effects of long-term cardiac resynchronization therapy: analysis by pressure-volume loops. Circulation 2006;113:1295-304.

[29] Baan J, van der Velde ET, de Bruin HG, Smeenk GJ, Koops J, van Dijk AD, et al. Continuous measurement of left ventricular volume in animals and humans by conductance catheter. Circulation 1984;70:812-23.

[30] Erb TO, Craig DM, Gaskin PM, Cheifetz IM, Resai BA, Sanders SP. Preload recruitable stroke work relationship in the right ventricle: simultaneous assessment using conductance catheter and sonomicrometry. Crit Care Med 2002;30:2535-41.

[31] Kuehne T, Yilmaz S, Steendijk P, Moore P, Groenink M, Saaed M, et al. Magnetic resonance imaging analysis of right ventricular pressure-volume loops: in vivo validation and clinical application in patients with pulmonary hypertension. Circulation 2004;110:2010-6.

[32] Schmidlin D, Aschkenasy S, Vogt PR, Schmidli J, Jenni R, Schmid ER. Left ventricular pressure-area relations as assessed by transoesophageal echocardiographic automated border detection: comparison with conductance catheter technique in cardiac surgical patients. Br J Anaesth 2000;85:379-88.

[33] Lambermont B, Gerard P, Detry O, Kolh P, Potty P, D'Orio V, et al. Correction of pressure waveforms recorded by fluid-filled catheter recording systems: a new method using a transfer equation. Acta Anaesthesiol Scand 1998;42:717-20.

[34] Bishop A, White P, Oldershaw P, Chaturvedi R, Brookes C, Redington A. Clinical application of the conductance catheter technique in the adult human right ventricle. Int J Cardiol 1997;58:211-21.

[35] Kameyama T, Asanoi H, Ishizaka S, Sasayama S. Ventricular load optimization by unloading therapy in patients with heart failure. J Am Coll Cardiol 1991;17:199-207. 